
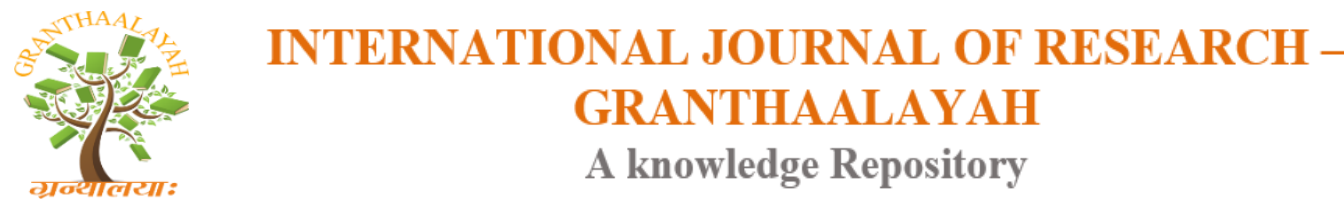

Science

\title{
REAL POWER LOSS REDUCTION \& VOLTAGE STABILITY AMPLIFICATION BY HYBRIDIZATION OF RESTARTED SIMULATED ANNEALING WITH PARTICLE SWARM OPTIMIZATION ALGORITHM
}

\author{
Dr. K. Lenin *1 \\ ${ }^{* 1}$ Professor, Department of EEE, Prasad V. Potluri Siddhartha Institute of Technology, Kanuru, \\ Vijayawada, Andhra Pradesh -520007, India
}

\begin{abstract}
This paper presents an algorithm for solving the multi-objective reactive power dispatch problem in a power system. Modal analysis of the system is used for static voltage stability assessment. Loss minimization and maximization of voltage stability margin are taken as the objectives. Generator terminal voltages, reactive power generation of the capacitor banks and tap changing transformer setting are taken as the optimization variables. Evolutionary algorithm and Swarm Intelligence algorithm (EA, SI), a part of Bio inspired optimization algorithm, have been widely used to solve numerous optimization problem in various science and engineering domains. Particle swarm optimization (PSO) is a population-based swarm intelligence algorithm that shares many similarities with evolutionary computation techniques. However, the PSO is driven by the simulation of a social psychological metaphor motivated by collective behaviors of bird and other social organisms instead of the survival of the fittest individual. The Simulated Annealing (SA) algorithm is a probabilistic hill-climbing technique that is based on the annealing/cooling process of metals. In total, most moves may be accepted at initial stages, but at the final stage only improving ones are likely to be allowed. This can help the procedure jump out of a local minimum. However, sometimes it is better to move back to a former solution that was significantly better rather than always moving from the current state. This process is called "restarting" of SA \& called as Restarted Simulated Annealing (RSA). In this paper we proposed a hybridized restarted simulated annealing particle swarm optimization (RSAPSO) technique to find global minima more efficiently and robustly. The proposed RSAPSO combines the global search ability of PSO and the local search ability of RSA, and offsets the weaknesses of each other. In order to evaluate the proposed algorithm, it has been tested on IEEE 30 bus system and compared to other reported algorithms.
\end{abstract}

Keywords: Global Search; Local Search; Simulated Annealing; Particle Swarm Optimization; Optimal Reactive Power; Transmission Loss.

Cite This Article: Dr. K. Lenin. (2018). "REAL POWER LOSS REDUCTION \& VOLTAGE STABILITY AMPLIFICATION BY HYBRIDIZATION OF RESTARTED SIMULATED ANNEALING WITH PARTICLE SWARM OPTIMIZATION ALGORITHM." International Journal of Research - Granthaalayah, 6(9), 246-258. https://doi.org/10.5281/zenodo.1442431. 


\section{Introduction}

Optimal reactive power dispatch problem is one of the difficult optimization problems in power systems. The sources of the reactive power are the generators, synchronous condensers, capacitors, static compensators and tap changing transformers. The problem that has to be solved in a reactive power optimization is to determine the required reactive generation at various locations so as to optimize the objective function. Here the reactive power dispatch problem involves best utilization of the existing generator bus voltage magnitudes, transformer tap setting and the output of reactive power sources so as to minimize the loss and to enhance the voltage stability of the system. It involves a non linear optimization problem. Various mathematical techniques have been adopted to solve this optimal reactive power dispatch problem. These include the gradient method [1, 2], Newton method [3] and linear programming [4-7]. The gradient and Newton methods suffer from the difficulty in handling inequality constraints. To apply linear programming, the input- output function is to be expressed as a set of linear functions which may lead to loss of accuracy. Recently Global Optimization techniques such as genetic algorithms have been proposed to solve the reactive power flow problem [8.9]. In recent years, the problem of voltage stability and voltage collapse has become a major concern in power system planning and operation. To enhance the voltage stability, voltage magnitudes alone will not be a reliable indicator of how far an operating point is from the collapse point [10]. The reactive power support and voltage problems are intrinsically related. Hence, this paper formulates the reactive power dispatch as a multi-objective optimization problem with loss minimization and maximization of static voltage stability margin (SVSM) as the objectives. Voltage stability evaluation using modal analysis [10] is used as the indicator of voltage stability. Particle swarm optimization (PSO) is a computational method that optimizes a problem by iteratively trying to improve a candidate solution with regard to a given measure of quality [11]. It is commonly known as meta heuristic method as it makes few or no assumptions about the problem being optimized and can search very large spaces of candidate solutions. PSO does not use the gradient of the problem being optimized, which means PSO does not require for the optimization problem to be differentiable as is required by classic optimization methods such as gradient descent and quasi-Newton methods. PSO can therefore also be used on optimization problems that are partially irregular, noisy, adaptive, etc [12]. PSO is widely applied in various fields. Lin et al. [13] proposed an immune PSO with functional link based neuro-fuzzy network for image backlight compensation. Fan et al. [14] integrates the PSO and entropy matching estimator to seek the optimal parameter of the generalized Gaussian distribution mixture model. Zahara et al. [15] used PSO to obtain the optimal thresholding of multilevel image segmentation. Zhang et al. [16] proposed an adaptive chaotic PSO for magnetic resonance brain image classification. Samanta et al. [17] integrated PSO to artificial neural networks (ANN) and support vector machine (SVM) for machinery fault detection, and demonstrated the results of PSO is superior to the ones of genetic algorithm. Zhang et al. [18] proposed a neural network by PSO for remote-sensing image classification. Unfortunately, PSO is easy to be trapped into local minima and its calculation efficiency is low. In the worst case, when the best solution found by the group and the particles are all located at the same local minimum, it is almost impossible for particles to jump out and do further searching due to the velocity update equation [19]. The reason lies in the fact that PSO is powerful of global search but weak on local search. Therefore, our strategy is to introduce in a local search which is applied during each update cycle. In this study, simulated annealing (SA) was chosen as the local search method. SA comes from annealing in metallurgy [20], a technique involving heating and controlled cooling of a material to increase the size of its crystals and reduce their defects [21]. The heat causes the atoms to become unstuck from 
their initial positions (a local minimum of the internal energy) and wander randomly through states of higher energy; the slow cooling gives them more chances of finding configurations with lower internal energy than the initial one [22]. Moreover, we introduced in the restarted simulated annealing (RSA) technique to improve the performance of SA. The hybrid algorithm combines both global search provided by PSO and local search provided by RSA [23]. The performance of (RSAPSO) has been evaluated in standard IEEE 30 bus test system and the results analysis shows that our proposed approach outperforms all approaches investigated in this paper. The performance of (RSAPSO) has been evaluated in standard IEEE 30 bus test system and the results analysis shows that our proposed approach outperforms all approaches investigated in this paper.

\section{Voltage Stability Evaluation}

\subsection{Modal Analysis for Voltage Stability Evaluation}

Modal analysis is one of the methods for voltage stability enhancement in power systems. In this method, voltage stability analysis is done by computing eigen values and right and left eigen vectors of a jacobian matrix. It identifies the critical areas of voltage stability and provides information about the best actions to be taken for the improvement of system stability enhancements. The linearized steady state system power flow equations are given by.

$\left[\begin{array}{c}\Delta \mathrm{P} \\ \Delta \mathrm{Q}\end{array}\right]=\left[\begin{array}{cc}\mathrm{J}_{\mathrm{p} \theta} & \mathrm{J}_{\mathrm{pv}} \\ \mathrm{J}_{\mathrm{q} \theta} & \mathrm{J}_{\mathrm{QV}}\end{array}\right]$

Where

$\Delta P=$ Incremental change in bus real power.

$\Delta Q=$ Incremental change in bus reactive

Power injection

$\Delta \theta=$ incremental change in bus voltage angle.

$\Delta V=$ Incremental change in bus voltage Magnitude

$\mathrm{J}_{\mathrm{p} \theta}, \mathrm{J}_{\mathrm{PV}}, \mathrm{J}_{\mathrm{Q} \theta}, \mathrm{J}_{\mathrm{QV}}$ jacobian matrix are the sub-matrixes of the System voltage stability is affected by both $\mathrm{P}$ and $\mathrm{Q}$. However at each operating point we keep $\mathrm{P}$ constant and evaluate voltage stability by considering incremental relationship between $\mathrm{Q}$ and $\mathrm{V}$.

To reduce (1), let $\Delta \mathrm{P}=0$, then.

$\Delta \mathrm{Q}=\left[\mathrm{J}_{\mathrm{QV}}-\mathrm{J}_{\mathrm{Q} \theta} \mathrm{J}_{\mathrm{P} \theta^{-1}} \mathrm{~J}_{\mathrm{PV}}\right] \Delta \mathrm{V}=\mathrm{J}_{\mathrm{R}} \Delta \mathrm{V}$

$\Delta \mathrm{V}=\mathrm{J}^{-1}-\Delta \mathrm{Q}$

Where

$\mathrm{J}_{\mathrm{R}}=\left(\mathrm{J}_{\mathrm{QV}}-\mathrm{J}_{\mathrm{Q} \theta} \mathrm{J}_{\mathrm{P \theta ^{-1 }}} \mathrm{JPV}\right)$

$\mathrm{J}_{\mathrm{R}}$ is called the reduced Jacobian matrix of the system. 


\subsection{Modes of Voltage Instability}

Voltage Stability characteristics of the system can be identified by computing the eigen values and eigen vectors

Let

$\mathrm{J}_{\mathrm{R}}=\xi \wedge \eta$

Where,

$\xi=$ right eigenvector matrix of $J_{R}$

$\eta=$ left eigenvector matrix of $J_{R}$

$\Lambda=$ diagonal eigenvalue matrix of $J_{R}$ and

$\mathrm{J}_{\mathrm{R}^{-1}}=\xi \wedge^{-1} \eta$

From (3) and (6), we have

$\Delta \mathrm{V}=\xi \wedge^{-1} \eta \Delta \mathrm{Q}$

Or

$\Delta \mathrm{V}=\sum_{\mathrm{I}} \frac{\xi_{\mathrm{i}} \eta_{\mathrm{i}}}{\lambda_{\mathrm{i}}} \Delta \mathrm{Q}$

Where $\xi_{i}$ is the ith column right eigenvector and $\eta$ the ith row left eigenvector of $J_{R}$.

$\lambda_{i}$ is the ith eigen value of $J_{R}$.

The ith modal reactive power variation is,

$\Delta \mathrm{Q}_{\mathrm{mi}}=\mathrm{K}_{\mathrm{i}} \xi_{\mathrm{i}}$

Where,

$\mathrm{K}_{\mathrm{i}}=\sum_{\mathrm{j}} \xi_{\mathrm{ij}}{ }^{2}-1$

Where

$\xi_{\mathrm{ji}}$ is the jth element of $\xi_{\mathrm{i}}$

The corresponding ith modal voltage variation is

$\Delta \mathrm{V}_{\mathrm{mi}}=\left[1 / \lambda_{\mathrm{i}}\right] \Delta \mathrm{Q}_{\mathrm{mi}}$

It is seen that, when the reactive power variation is along the direction of $\xi_{\mathrm{i}}$ the corresponding voltage variation is also along the same direction and magnitude is amplified by a factor which is equal to the magnitude of the inverse of the ith eigenvalue. In this sense, the magnitude of each eigenvalue $\lambda_{\mathrm{i}}$ determines the weakness of the corresponding modal voltage. The smaller the 
magnitude of $\lambda_{\mathrm{i}}$, the weaker will be the corresponding modal voltage. If $\left|\lambda_{\mathrm{i}}\right|=0$ the ith modal voltage will collapse because any change in that modal reactive power will cause infinite modal voltage variation.

In (8), let $\Delta \mathrm{Q}=\mathrm{e}_{\mathrm{k}}$ where $\mathrm{e}_{\mathrm{k}}$ has all its elements zero except the kth one being 1 . Then,

$$
\Delta \mathrm{V}=\sum_{\mathrm{i}} \frac{\mathrm{\eta}_{1 \mathrm{k}} \xi_{1}}{\lambda_{1}}
$$

$\eta_{1 \mathrm{k}} \quad \mathrm{k}$ th element of $\eta_{1}$

$\mathrm{V}-\mathrm{Q}$ sensitivity at bus $\mathrm{k}$

$$
\frac{\partial \mathrm{V}_{\mathrm{K}}}{\partial \mathrm{Q}_{\mathrm{K}}}=\sum_{\mathrm{i}} \frac{\eta_{1 \mathrm{k}} \xi_{1}}{\lambda_{1}}=\sum_{\mathrm{i}} \frac{\mathrm{P}_{\mathrm{ki}}}{\lambda_{1}}
$$

\section{Problem Formulation}

The objectives of the reactive power dispatch problem considered here is to minimize the system real power loss and maximize the static voltage stability margins (SVSM).

\subsection{Minimization of Real Power Loss}

It is aimed in this objective that minimizing of the real power loss (Ploss) in transmission lines of a power system. This is mathematically stated as follows.

$$
\mathrm{P}_{\text {loss }}=\sum_{\mathrm{k}=(\mathrm{i}, \mathrm{j})}^{\mathrm{n}} \mathrm{g}_{\mathrm{k}\left(\mathrm{V}_{\mathrm{i}}^{2}+\mathrm{V}_{\mathrm{j}}^{2}-2 \mathrm{~V}_{\mathrm{i}} \mathrm{V}_{\mathrm{j}} \cos \theta_{\mathrm{ij}}\right)}
$$

Where $\mathrm{n}$ is the number of transmission lines, $\mathrm{g}_{\mathrm{k}}$ is the conductance of branch $\mathrm{k}, \mathrm{V}_{\mathrm{i}}$ and $\mathrm{V}_{\mathrm{j}}$ are voltage magnitude at bus $i$ and bus $j$, and $\theta i j$ is the voltage angle difference between bus $i$ and bus j.

\subsection{Minimization of Voltage Deviation}

It is aimed in this objective that minimizing of the Deviations in voltage magnitudes (VD) at load buses. This is mathematically stated as follows.

Minimize $V D=\sum_{\mathrm{k}=1}^{\mathrm{nl}}\left|\mathrm{V}_{\mathrm{k}}-1.0\right|$

Where $\mathrm{nl}$ is the number of load busses and $\mathrm{V}_{\mathrm{k}}$ is the voltage magnitude at bus $\mathrm{k}$.

\subsection{System Constraints}

In the minimization process of objective functions, some problem constraints which one is equality and others are inequality had to be met. Objective functions are subjected to these constraints shown below. 
Load flow equality constraints:

$P_{G i}-P_{D i}-V_{i \sum_{j=1}^{n b} V_{j}}\left[\begin{array}{cc}G_{i j} & \cos \theta_{i j} \\ +B_{i j} & \sin \theta_{i j}\end{array}\right]=0, i=1,2 \ldots, n b$

$Q_{G i}-Q_{D i}-V_{i \sum_{j=1}^{n b} V_{j}}\left[\begin{array}{cc}G_{i j} & \cos \theta_{i j} \\ +B_{i j} & \sin \theta_{i j}\end{array}\right]=0, i=1,2 \ldots, n b$

Where, $n b$ is the number of buses, $P_{G}$ and $Q_{G}$ are the real and reactive power of the generator, $P_{D}$ and $Q_{D}$ are the real and reactive load of the generator, and $G_{i j}$ and $B_{i j}$ are the mutual conductance and susceptance between bus $i$ and bus $j$. Generator bus voltage $\left(V_{G i}\right)$ inequality constraint:

$V_{G i}^{\min } \leq V_{G i} \leq V_{G i}^{\max }, i \in n g$

Load bus voltage $\left(V_{L i}\right)$ inequality constraint:

$V_{L i}^{\min } \leq V_{L i} \leq V_{L i}^{\max }, i \in n l$

Switchable reactive power compensations $\left(Q_{C i}\right)$ inequality constraint:

$Q_{C i}^{\min } \leq Q_{C i} \leq Q_{C i}^{\max }, i \in n c$

Reactive power generation $\left(Q_{G i}\right)$ inequality constraint:

$Q_{G i}^{\min } \leq Q_{G i} \leq Q_{G i}^{\max }, i \in n g$

Transformers tap setting $\left(T_{i}\right)$ inequality constraint:

$T_{i}^{\min } \leq T_{i} \leq T_{i}^{\max }, i \in n t$

Transmission line flow $\left(\mathrm{S}_{\mathrm{Li}}\right)$ inequality constraint:

$S_{L i}^{\min } \leq S_{L i}^{\max }, i \in n l$

Where, nc, ng and nt are numbers of the switchable reactive power sources, generators and transformers.

\section{Particle Swarm Optimization}

PSO is a population based stochastic optimization technique, which simulates the social behaviour of a swarm of bird, flocking bees, and fish schooling. By randomly initializing the algorithm with candidate solutions, the PSO successfully leads to a global optimum. This is achieved by an iterative procedure based on the processes of movement and intelligence in an evolutionary system. 
The optimal algorithm of basic particle swarm: Supposing in the D-dimensional objects searching space, there is a community composed of $\mathrm{N}$ particle. The "I" particle represent a Ddimensional vector,

$X_{i}=\left(X_{i 1}, X_{i 2}, \ldots X_{i d}\right)$ It means that the "i" particle represents its position in this space. Every position of particle " $X$ " is a potential solution. If we put " $X$ " into objective function, we can know the adaptive value. We can know whether the " $x$ " is the optimal answer based on the adaptive value. The speed of particle is also a D-dimensional, it also recorded as $v_{i}=\left(v_{i 1}, v_{i 2}, \ldots v_{i d}\right)$. We record the particle I to the $\mathrm{h}$ times, the optimal position was $P_{i}=\left(P_{i 1}, P_{i 2}, \ldots P_{i d}\right)$. All the particles to the $\mathrm{h}$ times, the optimal position was $P_{g d}=\left(P_{i 1}, P_{i 2}, \ldots P_{i d}\right)$. The basic formulas are as follows:

$v_{i d}^{t+1}=w v_{i d}^{t}+c_{1} r_{1}^{t}\left(P_{i d}^{t}-x_{i d}^{t}\right)+c_{2} r_{2}^{t}\left(P_{g d}^{t}-x_{i d}^{t}\right)$

$x_{i d}^{t+1}=x_{i d}^{t}+v_{i d}^{t+1}$

Where, $\boldsymbol{c}_{\mathbf{1}} \& \boldsymbol{c}_{\mathbf{2}}$ : Speeding coefficient, adjusting the maxim step length that flying the best particle in whole situation and the individual best particle respectively. Appropriate $c_{1}$ and $c_{2}$ speed up the convergence and avoid falling into partial optimality $\mathbf{r}_{1} \& r_{2}$ : Random number between 0 and 1 , for controlling the weight of speed W: Inertia factor. It was oriented toward overall searching. The population of particles is then moved according to (24) and (25), and tends to cluster together from different directions. However, a maximum velocity $\mathrm{v}_{\max }$, should not be exceeded by any particle to keep the search within a meaningful solution space. The PSO algorithm runs through these processes iteratively until the termination criterion is satisfied [24].

\section{Restarted Simulated Annealing}

The SA algorithm is a probabilistic hill-climbing technique that is based on the annealing/cooling process of metals [25]. This annealing process occurs after the heat source is removed from a molten metal and its temperature starts to decrease. At each temperature level the energy of the metal molecules reduces, and the metal becomes more rigid. The procedure continues until the metal temperature has reached the surrounding ambient temperature, at which stage the energy has reached its lowest value and the metal is perfectly solid [26]. The SA procedure begins by generating an initial solution at random. At initial stages, a small random change is made in the current solution $X_{c}$. The new solution is called $X_{n}$. The perturbation depends on a temperate parameter $T$, and a scaling constant $\mathrm{k}$.

$\operatorname{pert}(T)=k \times T \times r_{3}$

Here $r_{3}$ is a random value between 0 and 1 with uniform distribution. The temperature $T$ decreases with each iteration of the algorithm, thus reducing the size of the perturbations as the search progresses. This mechanism produces large perturbation in the initial stages of the search and ensures that the resulting parameters are fine tuned towards the end of the optimization [27].

A move is made to the new solution $\mathrm{X}_{\mathrm{n}}$ if it has better energy $F$ or if the probability function has a higher value than a randomly generated number. Otherwise a new solution is generated, 
evaluated and compared again. The probability $p$ of accepting a new solution $\mathrm{X}_{\mathrm{n}}$ which called "Metropolis law" is given as follows:

$P=\left\{\begin{array}{rr}1 & \text { if } F\left(X_{n}\right)<F\left(X_{c}\right) \\ & \exp \left(\frac{F\left(X_{c}\right)-F\left(X_{n}\right)}{T}\right) \text { otherwise }\end{array}\right.$

In order to avoid getting trapped at local extrema points, the reduction rate of $T$ should be slow enough. In this study the following method to reduce the temperature has been used:

Here $T_{0}$ is the initial temperature, $\beta$ is the reduction constant, and $n$ is the number of iterations. In total, most worsening moves may be accepted at initial stages, but at the final stage only improving ones are likely to be allowed. This can help the procedure jump out of a local minimum. However, sometimes it is better to move back to a former solution that was significantly better rather than always moving from the current state. This process is called "restarting" of SA \& called as Restarted Simulated Annealing (RSA) [28]. To do this we set the temperature to a former value and restart the annealing schedule. The decision to restart can be based on several criteria, including whether a fixed number of steps had passed, whether the current energy being too high from the best obtained so far, or randomly restart. In this paper, we restart the SA when the current energy is too high from the best energy because it performs best among all criterions [29].

$T_{n}=T_{0} \times \beta^{n}$

\section{Hybrid RSAPSO Technique}

Traditional PSO algorithm suffers from getting trapped at the early stage. On the other side, RSA accepts a worse solution so it can escape from local point, resist earliness convergence, and increase the diversity of PSO [12]. Therefore, a new hybrid strategy was proposed which is referred to as RSAPSO. The proposed algorithm makes full use of the exploration ability both of PSO and of RSA and offsets the weaknesses of each other.

RSAPSO Algorithm for reactive power dispatch problem

Step 1 Initialize the population randomly;

Step 2 Evaluate each particle's fitness function $f$;

Step 3 Halve the population randomly: one half was updated by PSO as the formula (24) (25), the other half was updated by SA as the formula (26)(27)(28);

Step 4 Repeat Step 2 and Step 3 until the termination criteria were satisfied;

Step 5 Output the final results.

\section{Simulation Results}

The validity of the proposed hybridized restarted simulated annealing particle swarm optimization (RSAPSO) Algorithm is demonstrated on IEEE-30 bus system. The efficiency of the proposed Waterway Algorithm (WA) is demonstrated by testing it on standard IEEE-30 bus system. 6 generator buses, 24 load buses and 41 transmission lines of which four branches are (6-9), (6-10) , (4-12) and (28-27) - are with the tap setting transformers in standard IEEE-30 bus system. Lower 
voltage magnitude limits at all buses are 0.95 p.u. and the upper limits are 1.1 for all the PV buses, for PQ buses \& reference bus it is 1.05 p.u.. Comparisons of results are shown in Table 5. In Table 1 optimal values of the control variables are given.

Table 1: Results of RSAPSO - ORPD optimal control variables

\begin{tabular}{|l|l|}
\hline Control variables & Values of Variable setting \\
\hline V1 & 1.0431 \\
V2 & 1.0400 \\
V5 & 1.0401 \\
V8 & 1.0300 \\
V11 & 1.0030 \\
V13 & 1.0301 \\
T11 & 1.000 \\
T12 & 1.000 \\
T15 & 1.010 \\
T36 & 1.010 \\
Qc10 & 2 \\
Qc12 & 3 \\
Qc15 & 2 \\
Qc17 & 0 \\
Qc20 & 2 \\
Qc23 & 2 \\
Qc24 & 2 \\
Qc29 & 2 \\
Real power loss & 4.1022 \\
SVSM & 0.2478 \\
\hline
\end{tabular}

Table 2 indicates the optimal values of the control variables $\&$ there is no limit violations in state variables. Mainly static voltage stability margin (SVSM) has increased from 0.2478 to 0.2489. contingency analysis was conducted using the control variable setting obtained in case 1 and case 2 to determine the voltage security of the system. In Table 3 the Eigen values equivalents to the four critical contingencies are given. Result reveal about the Eigen value has been improved considerably for all contingencies in the second case.

Table 2: Results of RSAPSO -Voltage Stability Control Reactive Power Dispatch Optimal Control Variables

\begin{tabular}{|l|l|}
\hline Control Variables & Values of Variable Setting \\
\hline V1 & 1.0467 \\
V2 & 1.0484 \\
V5 & 1.0470 \\
V8 & 1.0300 \\
V11 & 1.0031 \\
V13 & 1.0320 \\
T11 & 0.090 \\
T12 & 0.090 \\
T15 & 0.090 \\
\hline
\end{tabular}




\begin{tabular}{|l|l|}
\hline T36 & 0.090 \\
Qc10 & 3 \\
Qc12 & 2 \\
Qc15 & 2 \\
Qc17 & 3 \\
Qc20 & 0 \\
Qc23 & 2 \\
Qc24 & 2 \\
Qc29 & 3 \\
Real power loss & 4.9896 \\
SVSM & 0.2489 \\
\hline
\end{tabular}

Table 3: Voltage Stability under Contingency State

\begin{tabular}{|l|l|l|l|}
\hline Sl.No & Contingency & $\begin{array}{l}\text { Optimal Reactive } \\
\text { Power } \\
\text { Dispatch Setting }\end{array}$ & $\begin{array}{l}\text { Voltage Stability Control Reactive Power } \\
\text { Dispatch } \\
\text { Setting }\end{array}$ \\
\hline 1 & $28-27$ & 0.1419 & 0.1424 \\
\hline 2 & $4-12$ & 0.1642 & 0.1651 \\
\hline 3 & $1-3$ & 0.1761 & 0.1764 \\
\hline 4 & $2-4$ & 0.2022 & 0.2052 \\
\hline
\end{tabular}

Table 4: Limit Violation Checking Of State Variables

\begin{tabular}{|l|l|l|l|l|}
\hline \multirow{2}{*}{$\begin{array}{l}\text { State } \\
\text { variables }\end{array}$} & \multicolumn{2}{|l|}{ limits } & $\begin{array}{l}\text { Optimal Reactive } \\
\text { Power } \\
\text { Dispatch Setting }\end{array}$ & $\begin{array}{l}\text { Voltage Stability Control Reactive } \\
\text { Power Dispatch } \\
\text { Setting }\end{array}$ \\
\hline Q1 & -20 & 152 & 1.3422 & -1.3269 \\
\hline Q2 & -20 & 61 & 8.9900 & 9.8232 \\
\hline Q5 & -15 & 49.92 & 25.920 & 26.001 \\
\hline Q8 & -10 & 63.52 & 38.8200 & 40.802 \\
\hline Q11 & -15 & 42 & 2.9300 & 5.002 \\
\hline Q13 & -15 & 48 & 8.1025 & 6.033 \\
\hline V3 & 0.95 & 1.05 & 1.0372 & 1.0392 \\
\hline V4 & 0.95 & 1.05 & 1.0307 & 1.0328 \\
\hline V6 & 0.95 & 1.05 & 1.0282 & 1.0298 \\
\hline V7 & 0.95 & 1.05 & 1.0101 & 1.0152 \\
\hline V9 & 0.95 & 1.05 & 1.0462 & 1.0412 \\
\hline V10 & 0.95 & 1.05 & 1.0482 & 1.0498 \\
\hline V12 & 0.95 & 1.05 & 1.0400 & 1.0466 \\
\hline V14 & 0.95 & 1.05 & 1.0474 & 1.0443 \\
\hline V15 & 0.95 & 1.05 & 1.0457 & 1.0413 \\
\hline V16 & 0.95 & 1.05 & 1.0426 & 1.0405 \\
\hline V17 & 0.95 & 1.05 & 1.0382 & 1.0396 \\
\hline V18 & 0.95 & 1.05 & 1.0392 & 1.0400 \\
\hline V19 & 0.95 & 1.05 & 1.0381 & 1.0394 \\
\hline V20 & 0.95 & 1.05 & 1.0112 & 1.0194 \\
\hline
\end{tabular}




\begin{tabular}{|l|l|l|l|l|}
\hline V21 & 0.95 & 1.05 & 1.0435 & 1.0243 \\
\hline V22 & 0.95 & 1.05 & 1.0448 & 1.0396 \\
\hline V23 & 0.95 & 1.05 & 1.0472 & 1.0372 \\
\hline V24 & 0.95 & 1.05 & 1.0484 & 1.0372 \\
\hline V25 & 0.95 & 1.05 & 1.0142 & 1.0192 \\
\hline V26 & 0.95 & 1.05 & 1.0494 & 1.0422 \\
\hline V27 & 0.95 & 1.05 & 1.0472 & 1.0452 \\
\hline V28 & 0.95 & 1.05 & 1.0243 & 1.0283 \\
\hline V29 & 0.95 & 1.05 & 1.0439 & 1.0419 \\
\hline V30 & 0.95 & 1.05 & 1.0418 & 1.0397 \\
\hline
\end{tabular}

Table 5: Comparison of Real Power Loss

\begin{tabular}{|l|l|}
\hline Method & Minimum loss \\
\hline Evolutionary programming [30] & 5.0159 \\
\hline Genetic algorithm [31] & 4.665 \\
\hline Real coded GA with Lindex as SVSM [32] & 4.568 \\
\hline Real coded genetic algorithm [33] & 4.5015 \\
\hline Proposed RSAPSO method & 4.1022 \\
\hline
\end{tabular}

\section{Conclusion}

In this paper a novel approach RSAPSO algorithm used to solve optimal reactive power dispatch problem, considering various generator constraints, has been successfully solved the problem. The performance of the proposed algorithm demonstrated through its voltage stability assessment by modal analysis is effective at various instants following system contingencies. Also this method has a good performance for voltage stability Enhancement of large, complex power system networks. The effectiveness of the proposed method is demonstrated on IEEE 30-bus system.

\section{References}

[1] O. Alsac, and B. Scott, "Optimal load flow with steady state security”, IEEE Transaction. PAS 1973, pp. 745-751.

[2] Lee K Y, Paru Y M, Oritz J L -A united approach to optimal real and reactive power dispatch, IEEE Transactions on power Apparatus and systems 1985: PAS-104 : 1147-1153

[3] A. Monticelli, M. V.F Pereira, and S. Granville, "Security constrained optimal power flow with post contingency corrective rescheduling", IEEE Transactions on Power Systems :PWRS-2, No. 1, pp.175-182.,1987.

[4] Deeb N, Shahidehpur S.M, Linear reactive power optimization in a large power network using the decomposition approach. IEEE Transactions on power system 1990: 5(2): 428-435

[5] E. Hobson, 'Network consrained reactive power control using linear programming, 'IEEE Transactions on power systems PAS -99 (4), pp 868-877, 1980

[6] K.Y Lee, Y.M Park, and J.L Oritz, "Fuel -cost optimization for both real and reactive power dispatches", IEE Proc; 131C, (3), pp.85-93.

[7] M.K. Mangoli, and K.Y. Lee, "Optimal real and reactive power control using linear programming", Electr.Power Syst.Res, Vol.26, pp.1-10,1993.

[8] S.R.Paranjothi ,and K.Anburaja, "Optimal power flow using refined genetic algorithm", Electr.Power Compon.Syst, Vol. 30, 1055-1063,2002. 
[9] D. Devaraj, and B. Yeganarayana, "Genetic algorithm based optimal power flow for security enhancement", IEE proc-Generation.Transmission and. Distribution; 152, 6 November 2005.

[10] C.A. Canizares, A.C.Z.de Souza and V.H. Quintana, "Comparison of performance indices for detection of proximity to voltage collapse," vol. 11. no.3, pp.1441-1450, Aug 1996.

[11] M.S. Arumugam, M.V.C. Rao, A.W.C. Tan, A novel and effective particle swarm optimization like algorithm with extrapolation technique, Applied Soft Computing, 9 (2009) 308-320.

[12] Y. Zhang, J. Yan, G. Wei, L. Wu, Find multi-objective paths in stochastic networks via chaotic immune PSO, Expert Systems with Applications, 37 (2010) 1911-1919.

[13] C.-J. Lin, Y.-C. Liu, Image backlight compensation using neurofuzzy networks with immune particle swarm optimization, Expert Systems with Applications, 36 (2009) 5212-5220.

[14] S.-K.S. Fan, Y. Lin, C.-C. Wu, Image thresholding using a novel estimation method in generalized Gaussian distribution mixture modeling, Neurocomputing, 72 (2008) 500-512.

[15] E. Zahara, S.-K.S. Fan, D.-M. Tsai, Optimal multi-thresholding using a hybrid optimization approach, Pattern Recognition Letters, 26 (2005) 1082-1095.

[16] Y. Zhang, S. Wang, L. Wu, A Novel Method for Magnetic Resonance Brain Image Classification based on Adaptive Chaotic PSO, Progress in Electromagnetics Research, 109 (2010) 325-343.

[17] B. Samanta, C. Nataraj, Use of particle swarm optimization for machinery fault detection, Engineering Applications of Artificial Intelligence, 22 (2009) 308-316.

[18] Y. Zhang, S. Wang, L. Wu, PSONN used for Remote-Sensing Image Classification, Journal of Computational Information Systems, 6 (2010) 4417- 4425.

[19] V. Kumar, P. Balasubramanian, Kinetic parameter estimation in hydrocracking using hybrid particle swarm optimization, Fuel, 88 (2009) 2171-2180.

[20] A. Nakib, H. Oulhadj, P. Siarry, Image histogram thresholding based on multiobjective optimization, Signal Processing, 87 (2007) 2516-2534.

[21] H. Mohamadi, J. Habibi, M.S. Abadeh, H. Saadi, Data mining with a simulated annealing based fuzzy classification system, Pattern Recognition, 41 (2008) 1824-1833.

[22] S. Rubenthaler, T. Rydén, M. Wiktorsson, Fast simulated annealing in with an application to maximum likelihood estimation in state-space models, Stochastic Processes and their Applications, 119 (2009) 1912- 1931.

[23] Y. Zhang, S. Wang, L. Wu, Y. Huo, Artificial Immune System for Protein folding model, Journal of Convergence Information Technology, 6 (2011) 55-61.

[24] A. Mahmood, Replicating web contents using a hybrid particle swarm optimization, Information Processing \& Management, 46 (2010) 170-179.

[25] E. Cosola, K. Genovese, L. Lamberti, C. Pappalettere, A general framework for identification of hyper-elastic membranes with moiré techniques and multi-point simulated annealing, International Journal of Solids and Structures, 45 (2008) 6074-6099.

[26] E.W. McGookin, D.J. Murray-Smith, Submarine manoeuvring controllers' optimisation using simulated annealing and genetic algorithms, Control Engineering Practice, 14 (2006) 1-15.

[27] Y. Zhang, L. Wu, N. Naggaz, S. Wang, G. Wei, Remote-sensing Image Classification Based on an Improved Probabilistic Neural Network, Sensors, 9 (2009) 7516-7539.

[28] N. Cretu, M.-I. Pop, Acoustic behavior design with simulated annealing, Computational Materials Science, 44 (2009) 1312-1318.

[29] I.A. Gheyas, L.S. Smith, Feature subset selection in large dimensionality domains, Pattern Recognition, 43 (2010) 5-13.

[30] Wu Q H, Ma J T. Power system optimal reactive power dispatch using evolutionary programming. IEEE Transactions on power systems 1995; 10(3): 1243-1248.

[31] S. Durairaj, D. Devaraj, P.S. Kannan,' Genetic algorithm applications to optimal reactive power dispatch with voltage stability enhancement', IE(I) Journal-EL Vol 87,September 2006.

[32] D. Devaraj,' Improved genetic algorithm for multi - objective reactive power dispatch problem' European Transactions on electrical power 2007; 17: 569-581. 
[33] P. Aruna Jeyanthy and Dr. D. Devaraj "Optimal Reactive Power Dispatch for Voltage Stability Enhancement Using Real Coded Genetic Algorithm" International Journal of Computer and Electrical Engineering, Vol. 2, No. 4, August, 2010 1793-8163.

*Corresponding author.

E-mail address: gklenin@ gmail.com 